\title{
APOM and high-density lipoprotein cholesterol are associated with lung function and per cent emphysema
}

\author{
Kristin M. Burkart, Ani Manichaikul, Jemma B. Wilk, Firas S. Ahmed, \\ Gregory L. Burke, Paul Enright, Nadia N. Hansel, Demondes Haynes, \\ Susan R. Heckbert, Eric A. Hoffman, Joel D. Kaufman, Jun Kurai, Laura Loehr, \\ Stephanie J. London, Yang Meng, George T. O'Connor, Elizabeth Oelsner, \\ Marcy Petrini, Tess D. Pottinger, Charles A. Powell, Susan Redline, \\ Jerome I. Rotter, Lewis J. Smith, María Soler Artigas, Martin D. Tobin, \\ Michael Y. Tsai, Karol Watson, Wendy White, Taylor R. Young, Stephen S. Rich \\ and R. Graham Barr
}

Affiliations: For a full list of the authors' affiliations please see the Acknowledgements.

Correspondence: R.G. Barr, Columbia University Medical Center, 630 West 168th Street, PH 9 East - Room 105, New York, NY 10032, USA. E-mail: rgb9acolumbia.edu

ABSTRACT Chronic obstructive pulmonary disease (COPD) is linked to cardiovascular disease; however, there are few studies on the associations of cardiovascular genes with COPD.

We assessed the association of lung function with 2100 genes selected for cardiovascular diseases among 20077 European-Americans and 6900 African-Americans. We performed replication of significant loci in the other racial group and an independent consortium of Europeans, tested the associations of significant loci with per cent emphysema and examined gene expression in an independent sample. We then tested the association of a related lipid biomarker with forced expiratory volume in $1 \mathrm{~s}$ (FEV1)/forced vital capacity (FVC) ratio and per cent emphysema.

We identified one new polymorphism for FEV1/FVC ( $r$ 805301) in European-Americans $\left(\mathrm{p}=1.3 \times 10^{-6}\right)$ and a second (rs707974) in the combined European-American and African-American analysis $\left(\mathrm{p}=1.38 \times 10^{-7}\right)$. Both single-nucleotide polymorphisms (SNPs) flank the gene for apolipoprotein $\mathrm{M}$ $(A P O M)$, a component of high-density lipoprotein (HDL) cholesterol. Both were replicated in an independent cohort. SNPs in a second gene related to apolipoprotein M and HDL, PCSK9, were associated with FEV1/FVC ratio among African-Americans. rs707974 was associated with per cent emphysema among European-Americans and African-Americans and APOM expression was related to FEV1/FVC ratio and per cent emphysema. Higher HDL levels were associated with lower FEV1/FVC ratio and greater per cent emphysema.

These findings suggest a novel role for the apolipoprotein M/HDL pathway in the pathogenesis of COPD and emphysema.

@ERSpublications

Findings from this study suggest a novel effect of the APOM/HDL pathway in the pathogenesis of COPD and emphysema http://ow.ly/t8Ao9

This article has supplementary material available from www.erj.ersjournals.com

Received: Sept 172012 | Accepted after revision: July 012013 | First published online: July 302013

Conflict of interest: Disclosures can be found alongside the online version of this article at www.erj.ersjournals.com 


\section{Introduction}

Chronic obstructive pulmonary disease (COPD) is a leading cause of death globally [1] and is characterised by persistent airflow obstruction [2,3]. Emphysema is defined anatomically by permanent enlargement of airspaces distal to terminal bronchioles with destruction of alveolar walls [4].

Familial studies suggest a genetic influence on COPD [5-8]. Recent genome-wide association studies (GWAS) have identified loci associated with the ratio of forced expiratory volume in $1 \mathrm{~s}$ (FEV1) to forced vital capacity (FVC) and COPD among participants of European ancestry [9-14]. Many of these genes have been shown to influence susceptibility to COPD [15-17]; however, they explained little more than 3\% of the variance in lung function.

Emphysema also has a familial predisposition [18]. However, understanding of the genetic basis for emphysema, beyond $\alpha_{1}$-antitrypsin deficiency, is more limited. One GWAS identified one genetic locus for radiologist-defined emphysema on computed tomography (CT) [19] and a second identified genetic loci in $\alpha$-mannosidase-related genes for quantitatively assessed emphysema [20]. Candidate gene association studies have identified additional genes for emphysema [21-26].

Complimentary genotyping strategies to better delineate the genetic basis of COPD and emphysema are therefore warranted. One such strategy is a "gene-centric" genotyping chip, which includes a large panel of candidate genes and often better gene coverage than GWAS chips. No such chips have been designed specifically for lung disease; however, the Institute of Translational Medicine and Therapeutics/Broad/ Candidate-gene Association Resource (CARe) Consortium (IBC) chip [27] includes 2100 candidate genes primarily selected for cardiovascular disease.

Respiratory and cardiac function are tightly linked at cellular [28, 29], physiological [30], structural [31] and anatomical levels. For example, endothelial dysfunction is implicated in the pathogenesis of atherosclerosis [32] and emphysema in animal models [33-36] and humans [37], the latter via ceramidemediated endothelial cell apoptosis [38-40]. High-density lipoprotein (HDL) may also be relevant to COPD and emphysema, as HDL increases in vitro ceramide levels [41]. HDL levels and function are affected by apolipoprotein M [42-44].

We examined associations of FEV1/FVC ratio on the IBC chip in European-American and AfricanAmerican participants in the CARe consortium [45]. Findings were replicated in the SpiroMeta consortium [12]. We performed additional analyses of identified genes with the percentage of emphysema-like lung (per cent emphysema), gene expression and HDL with lung function and per cent emphysema in the MultiEthnic Study of Atherosclerosis (MESA) Single Nucleotide Polymorphism (SNP) Health Association Resource (SHARe) and MESA COPD studies.

\section{Methods}

Study samples

The appropriate institutional review boards approved the study protocols and written informed consent was obtained from all participants.

Support statement: National Institutes of Health (NIH)/National Heart, Lung, and Blood Institute (NHLBI) grants RC1HL100543, R01-HL077612 and R01-HL093081, in addition to the following. Atherosclerosis Risk in Communities: HHSN268201100005C, HHSN268201100006C, HHSN268201100007C, HHSN268201100008C, HHSN268201100009C, HHSN268201100010C, HHSN268201100011C, HHSN268201100012C, R01HL087641, R01HL59367, R01HL086694, NIH/National Human Genome Research Institute contract U01HG004402, NIH contract HHSN268200625226C, N01HC-55015, N01-HC-55016, N01-HC-55021, N01-HC-55019, N01-HC-55020, N01-HC-55017 and N01-HC-55018, and UL1RR025005, a component of the NIH and NIH Roadmap for Medical Research; Broad Institute: N01-HC-65226; Coronary Artery Risk Development in Young Adults (CARDIA): N01-HC-48047, N01-HC-48048, N01-HC-48049, N01HC-48050, N01-HC-95095, N01-HC-45204, N01-HC-45205, N01-HC-05187, N01-HC-45134 and N01-HC-95100; Cleveland Family Study (CFS): RO1 HL46380-01-16; Cardiovascular Health Study (CHS): CHS research was supported by NHLBI contracts N01-HC-85239, N01-HC-85079, N01-HC-85080, N01-HC-85081, N01-HC-85082, N01-HC-85083, N01-HC-85084, N01-HC-85085, N01-HC-85086, N01-HC-35129, N01 HC-15103, N01-HC-75150, N01-HC-45133, N01 HC-55222, U01 HL080295, AG-023629, AG-15928, AG-20098 and AG-027058 with additional contribution from National Institute of Neurological Diseases and the National Institute on Aging; Framingham Heart Study (FHS): N01HC-25195; Jackson Heart Study (JHS): N01-HC-95170, N01-HC-95171 and N01-HC-95172; Multi-Ethnic Study of Atherosclerosis (MESA): N01-HC-95159, N01-HC-95160, N01-HC-95161, N01-HC-95162, N01-HC-95163, N01-HC95164, N01-HC-95165, N01-HC-95166, N01-HC-95167, N01-HC-95168, N01-HC-95169, R01-HL093081, RR-024156, R01-HL-071051, R01-HL-071205, R01-HL-071250, R01-HL-071251, R01-HL-071252, R01-HL-071258, R01-HL-071259 and Environmental Protection Agency (EPA) grant RD831697; SpiroMeta: M.D. Tobin holds a Medical Research Council Senior Clinical Fellowship (G0902313) and research undertaken by M.D. Tobin was part-funded by the National Institute for Health Research. S.J. London is supported by the Division of Intramural Research, National Institute of Environmental Health Sciences, NIH and the Department of Health and Human Services. 
Analyses of lung function

The association of genes and lung function were assessed in the seven CARe cohorts that measured spirometry: Atherosclerosis Risk in Communities (ARIC), Coronary Artery Risk Development in Young Adults (CARDIA), Cleveland Family Study (CFS), Cardiovascular Health Study (CHS), Framingham Heart Study (FHS), Jackson Heart Study (JHS) and the subset of MESA with spirometry. These cohorts have been described previously [46-55] and are summarised in the online supplementary material. Exclusion criteria were lack of valid spirometric or genetic data, age $<23$ years and a restrictive pattern of spirometry, defined as FVC less than the lower limit of normal [56] and FEV1/FVC ratio $>0.70$.

\section{Replication of lung function SNPS}

Replication of FEV1/FVC ratio was performed in the SpiroMeta consortium, a large independent sample of 14 GWAS studies [12]. Replication for airflow obstruction was performed using publically available data from the SpiroMeta and Cohorts for Heart and Aging Research in Genomic Epidemiology (CHARGE) consortia [10], which partly overlap with European-American participants in the CARe consortium. Details are provided in the online supplementary material.

\section{Analyses of per cent emphysema}

Per cent emphysema was examined in all participants in MESA SHARe, which comprises all participants who consented to genetic analyses in the MESA [46], MESA Family [57] and MESA Air Pollution [58] studies. Spirometry was not required.

Gene expression analyses

mRNA expression was examined in peripheral blood mononuclear cells in the MESA COPD Study, an independent sample described in the online supplementary material.

\section{Phenotypic measures \\ Spirometry}

Pre-bronchodilator spirometry was performed by trained and certified spirometry technicians in accordance with American Thoracic Society guidelines. Spirometry methods and equipment were highly standardised and in some cases identical across cohorts, as described in the online supplementary material.

\section{Per cent emphysema}

Per cent emphysema was assessed in MESA SHARe on lung fields of cardiac CT scans, which image $\sim 70 \%$ of lung volume from the carina to the lung bases, at a single centre by trained readers, as previously described, and validated compared to full-lung scans [59]. Per cent emphysema was defined as percentage of total voxels in the lung $<-950 \mathrm{HU}$. The MESA COPD study used the same approach on full-lung scans using Apollo software (Vida Diagnostics, Coralville, IA, USA).

HDL

HDL was measured in EDTA plasma using the cholesterol oxidase method (Roche Diagnostics Corporation, Indianapolis, IN, USA) after precipitation of non-HDL cholesterol with magnesium/dextran [60].

\section{Genotyping}

All CARe participants were genotyped using the IBC Illumina iSELECT array, a 50000 gene-centric SNP array [27]. All genotyping was performed at a single centre. Quality control methods are described in the online supplementary material.

MESA SHARe participants were genotyped using the Affymetrix Genome-Wide Human SNP Array 6.0 platform (Affymetrix, Santa Clara, CA, USA) at a single centre.

\section{Statistical analyses}

Analyses of candidate genes with FEV1/FVC ratio employed linear regression, stratified by race and adjusted for age, age ${ }^{2}$, height, height ${ }^{2}$, sex, smoking status, pack-years, pack-years ${ }^{2}$, site (if applicable) and the first 10 principal components for ancestry. Association testing of rank-normalised residuals was performed under an additive genetic model [61-63].

Cohort-specific association results were meta-analysed, again stratified by race, using inverse variance weighting in METAL [64] with cohort-specific and overall genomic control. A priori, we planned to replicate our top loci identified among European-Americans in the African-American cohorts and vice versa as distinct cohorts. Race-specific results were then meta-analysed in METAL [64] for the combined European-American and African-American analyses. The Bonferroni-adjusted thresholds for statistical 
significance in CARe were $1.31 \times 10^{-6}$ in European-Americans and $1.13 \times 10^{-6}$ in African-Americans and combined analyses, which are exceedingly conservative for the IBC chip.

Analyses for log-transformed per cent emphysema used the same analytical approach supplemented with a linear mixed effects model for family-based-data [62], and adjustment for age, sex, site, scanner, height, weight, tube current, cigarettes per day, pack-years, asthma and principal components for ancestry.

Analysis details for gene expression and HDL association studies with lung function and per cent emphysema are provided in the online supplementary material.

To address multiple comparisons, we considered analysis of per cent emphysema to be analogous to a modified Holm's procedure [65] on the pathway of apolipoprotein M. We hypothesised that SNPs rs805301 and rs707974 are in linkage disequilibrium with the causative APOM (apolipoprotein $\mathrm{M}$ ) variant that affects per cent emphysema, which affects FEV1/FVC ratio, thus the Holm-Bonferonni corrected threshold for statistical significance for subsequent analyses was set at 0.025 .

\section{Results}

The mean \pm SD age of the 26977 CARe participants with spirometry was $54 \pm 13$ years, $52 \%$ were eversmokers, with a median of 20 pack-years. Additional characteristics of the 20077 European-American and 6900 African-American participants are shown by cohort and race in table 1.

\section{Association study of 2100 candidate genes with lung function in CARe}

Among European-Americans, we identified one new SNP (rs805301) for FEV1/FVC ratio (fig. 1a). Among African-Americans, no SNPs were significantly associated with FEV1/FVC ratio using the Bonferroni cutoff; however, three SNPs were significant with the less conservative cut-off $\left(\mathrm{p}<1 \times 10^{-5}\right)$ (fig. $\left.1 \mathrm{~b}\right)$. In the combined European-American and African-American analysis, we identified a second new SNP (rs707974) for FEV1/FVC ratio (fig. 1c and table 2).

The new SNP (rs805301) identified in European-Americans was selected for the IBC chip as a variant in $A P O M$ based upon Genome Build 36 and is annotated in BAG6 (B-cell lymphoma-2-associated athanogene 6), which is the upstream flanking gene of $A P O M$, on Genome Build 37.3. It replicated among AfricanAmericans $(\mathrm{p}=0.036)$ and remained significant in the combined meta-analysis (table 2). The risk allele $(C)$ was associated with a decrease in FEV1/FVC ratio in both racial groups.

The new SNP identified in the combined European-American and African-American analysis, rs707974, was the second most significant SNP in African-Americans (table 2) and would have been significant with a less stringent Bonferroni cut-off. It was also selected as an APOM variant and is now annotated in GPANK1 (G patch domain and ankyrin repeats 1), the downstream flanking gene of APOM separated by an openreading frame, C6orf47. It was not significant for FEV1/FVC ratio in European-Americans $\left(\mathrm{p}=2.84 \times 10^{-5}\right)$.

The SNPs rs805301 and rs707974 were not in high linkage disequilibrium in European-Americans or African-Americans $\left(r^{2}=0.07\right.$ and $r^{2}=0.03$, respectively), suggesting that they are separate loci (fig. 2). They were also not in high linkage disequilibrium with the previously described AGER (advanced glycosylation end product receptor) SNP rs2070600 in European-Americans $\left(r^{2}=0.035\right.$ and $r^{2}=0.37$, respectively) [ 12 , 13]. In addition, rs805301 remained associated with FEV1/FVC after adjustment for rs 2070600 $\left(\mathrm{p}=6.82 \times 10^{-4}\right)$ and $\mathrm{rs} 2070600$ was only nominally associated with FEV1/FVC among African-Americans $(\mathrm{p}=0.009)$. These findings suggest that associations of rs805301and rs707974 with lung function are unrelated to $A G E R$.

Sensitivity analyses restricted to participants free of clinical cardiovascular disease, aged $\leqslant 55$ years and free of asthma yielded similar results, as did analyses additionally adjusted for diabetes, hypertension and asthma (online supplementary material). Analyses stratified by smoking status yielded similar results (online supplementary material).

The other top loci in African-Americans were in NFKBIA (nuclear factor of $\kappa$ light polypeptide gene enhancer in B-cells inhibitor- $\alpha$ ) and PCSK9 (proprotein convertase subtilisin/kexin type 9) (table 2). PCSK9 is related to apolipoprotein M [67] and six of the top 30 SNPs for FEV1/FVC in African-Americans were in PCSK9 (fig. 2). SNPs in neither gene replicated in European-Americans. Regional association plots for additional loci are displayed in online supplementary figure S3.

Results for the FEV1 are displayed in online supplementary figures S4, S5 and S6. Top SNPs associated with $\mathrm{FEV} 1 / \mathrm{FVC}$ ratio and FEV1 are presented in online supplementary tables S1 and S2, respectively. 


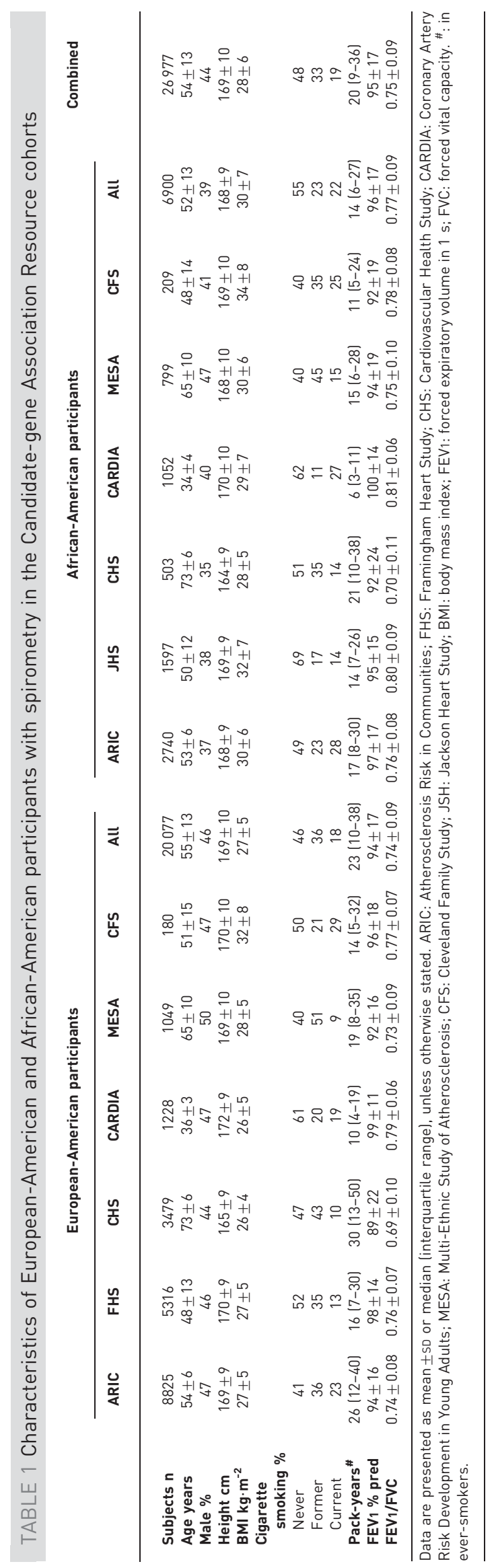



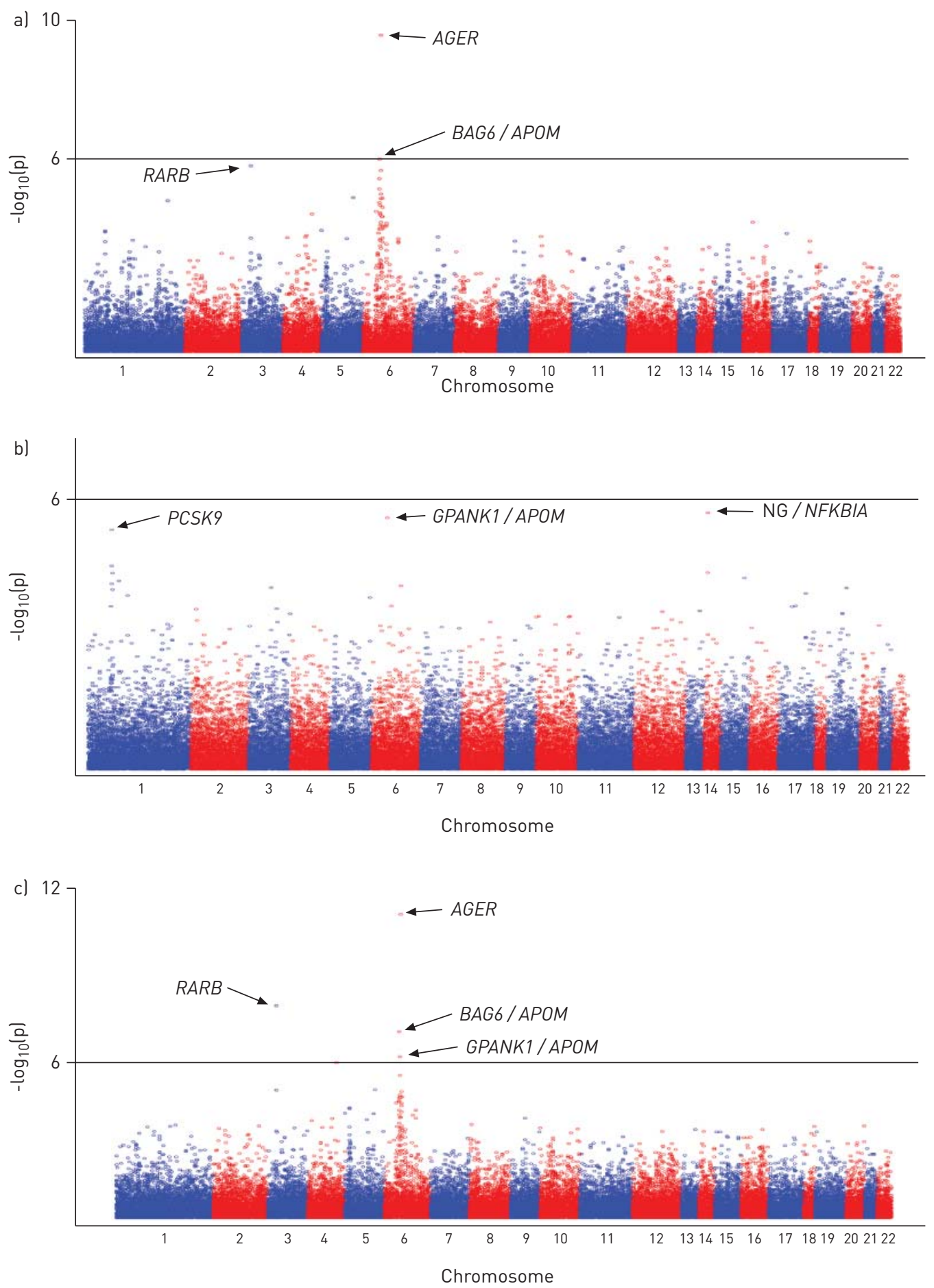

FIGURE 1 Manhattan plots of association results for forced expiratory volume in $1 \mathrm{~s}$ (FEV1)/forced vital capacity (FVC) ratio stratified by race and combined. Manhattan plots are ordered by the chromosome position of the association results for $\mathrm{FEV}_{1} / \mathrm{FVC}$ ratio. The top loci are labelled with arrows. a) Meta-analysis of 38294 single nucleotide polymorphisms (SNPs) among 20077 European-American participants; b) meta-analysis of 44416 SNPs among 6900 African-American participants; c) meta-analysis of SNPs among combined European-American and African-American participants. The solid black lines represent $1 \times 10^{-6}$. AGER: advanced glycosylation end product receptor; RARB: retinoic acid receptor- $\beta$; BAG6: B-cell lymphoma-2-associated athanogene 6; APOM: apolipoprotein M; PCSK9: proprotein convertase subtilisin/ kexin type 9; GPANK1: G patch domain and ankyrin repeats 1; NG: near gene; NFKBIA: nuclear factor of $\kappa$ light polypeptide gene enhancer in B-cells inhibitor- $\alpha$. 


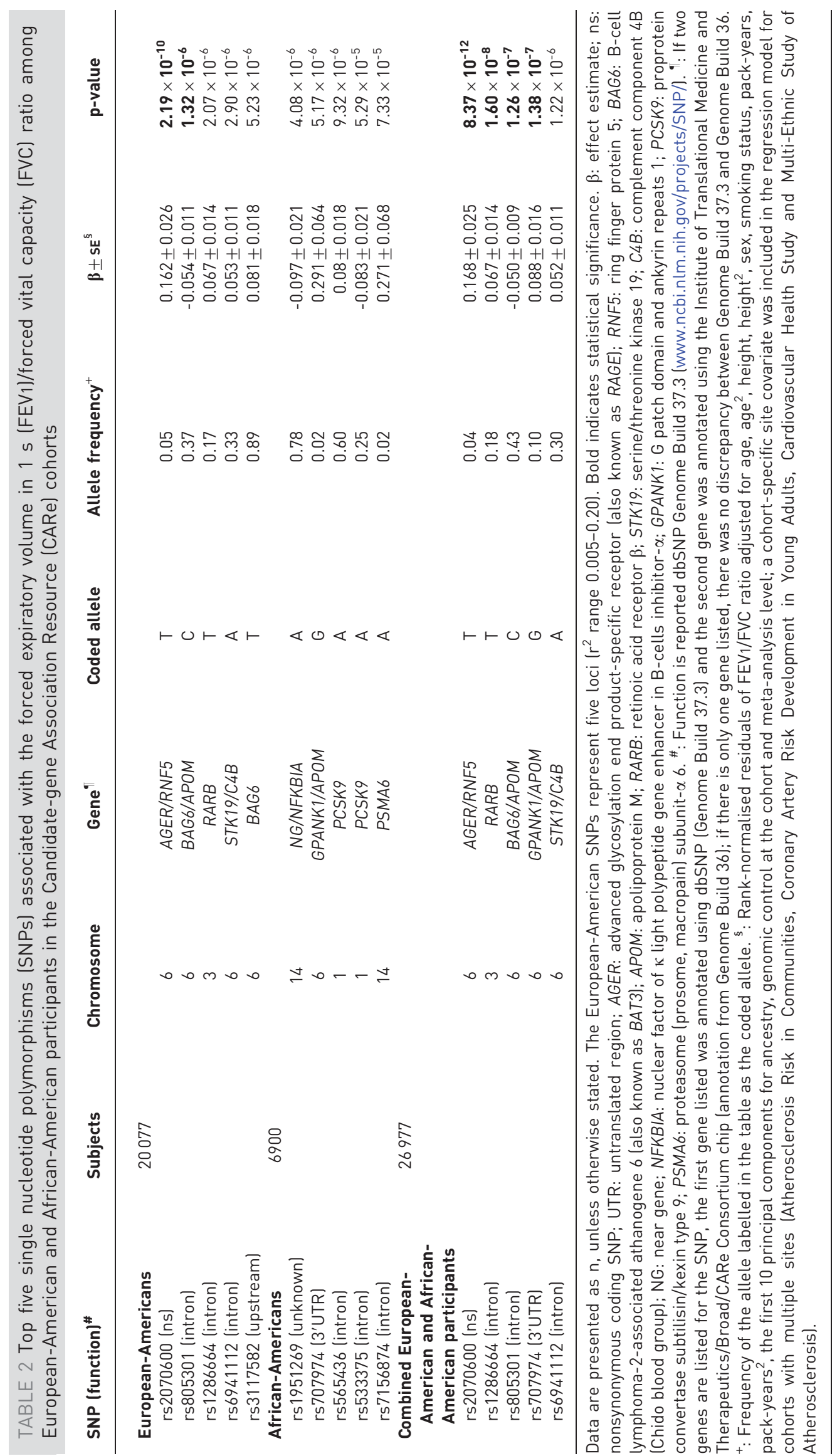



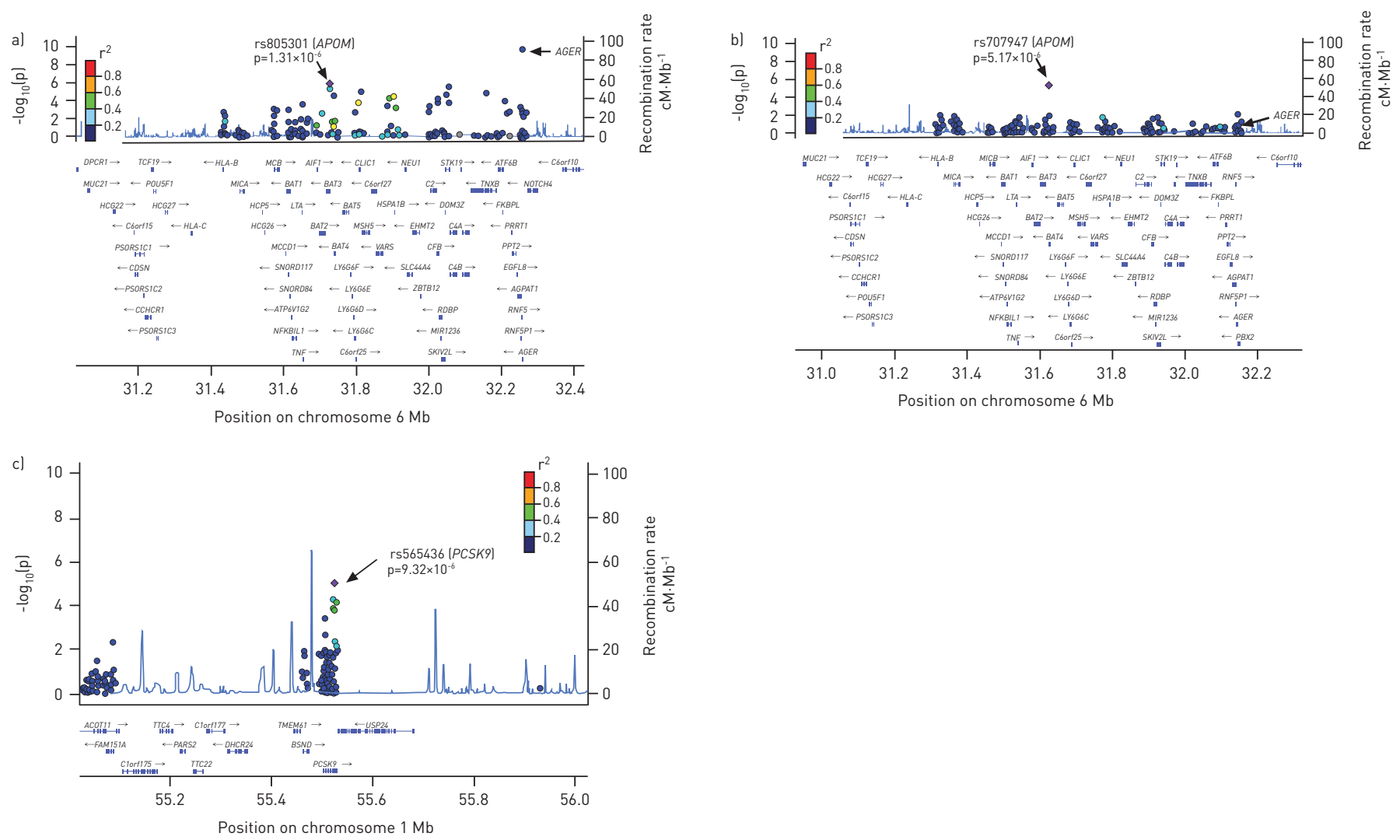

FIGURE 2 Regional association plots of top single nucleotide polymorphisms (SNPs) flanking the APOM (apolipoprotein M) (rs805301 and rs707974) and PCSK9 (proprotein convertase subtilisin/kexin type 9) SNPs for forced expiratory volume in 1 s/forced vital capacity ratio in a) European-American participants, b) African-American participants (chromosome 6), and c) African-American participants (chromosome 1). The selected SNPs with the lowest p-value are illustrated by the purple diamond. The correlations $\left(\mathrm{r}^{2}\right)$ of surrounding SNPs in the region are indicated by the colours shown on the graph. For the SNPs flanking APOM (rs805301 and rs707974), a 600-kb flanking size was selected to include the AGER (advanced glycosylation end product-specific receptor) SNP on the plot, whereas a flanking size of $500 \mathrm{~kb}$ was selected for the PCSK9 SNP. Plots were generated using LocusZoom [66]. The genome builds/linkage disequilibrium populations implemented were hg18/HapMap Phase II CEU and hg/19 1000 Genomes Nov 2010 AFR for European-American and for AfricanAmerican participants, respectively.

\section{Replication of SNPs flanking APOM in SpiroMeta}

Both rs805301 and rs707974 replicated for FEV1/FVC ratio in 20288 European participants in the SpiroMeta consortium in a consistent direction $(\beta=-0.03, p=0.02$ and $\beta=0.05, p=0.02$, respectively).

We reviewed publically available results from the SpiroMeta-CHARGE GWAS meta-analysis of airflow obstruction [10]. SNP rs805301 was associated with airflow obstruction $(p=0.004)$ and rs707974 was nominally associated with airflow obstruction in individuals without asthma $(\mathrm{p}=0.026)$ (online supplementary material).

\section{Association of SNPs flanking APOM with per cent emphysema in MESA}

SNP rs707974 was significantly associated with per cent emphysema among 2552 European-Americans and 2483 African-Americans $\left(\mathrm{p}=4.74 \times 10^{-4}\right.$ and $\mathrm{p}=0.009$, respectively) and in combined analyses $\left(\mathrm{p}=1.67 \times 10^{-5}\right) \quad($ table 3$)$ in MESA. The characteristics of these participants are shown in online supplementary table S3. The direction of the association of rs707974 with per cent emphysema and lung function was consistent: risk allele (A) was associated with greater per cent emphysema and a lower FEV1/ FVC ratio.

The association with per cent emphysema persisted in an independent sample of 1138 European-American and 1563 African-American MESA participants who did not have spirometry measures and who were therefore excluded from the lung function analysis $(\mathrm{p}=0.02$ and $\mathrm{p}=0.003$, respectively). Additional adjustment for socioeconomic status yielded similar results, whereas restriction to 418 European-Americans and 209 African-Americans with FEV1/FVC ratio $<0.70$ yielded nonsignificant results; however, the effect size was greater in African-Americans and similar in European-Americans in these groups compared to the 
overall MESA sample (online supplementary material). SNP rs805301 was not significantly associated with per cent emphysema.

PCSK9 was nominally associated with per cent emphysema in European-Americans $(\mathrm{p}=0.04)$ but not African-Americans. AGER SNP rs2070600 was significantly associated with per cent emphysema among European-Americans and African-Americans $\left(\mathrm{p}=2.54 \times 10^{-4}\right.$ and $\mathrm{p}=0.001$, respectively).

\section{Gene expression of SNPs flanking APOM in MESA COPD}

APOM expression was significantly inversely associated with FEV1/FVC ratio (table 4) in an independent sample of 101 participants in the MESA COPD Study, the characteristics of which are described in the online supplementary material. We secondarily examined expression of GPANK1, BAG6 and PCSK9. GPANK1 expression was associated with FEV1/FVC ratio $(\beta=-0.096,95 \%$ CI $-0.175--0.017 ; \mathrm{p}=0.02)$, whereas BAG6 and PCSK9 expression were not associated with FEV1/FVC ratio.

APOM expression was positively associated with per cent emphysema in minimally adjusted models and after adjustment for BAG6 (table 4). BAG6 expression was not associated with per cent emphysema, except after adjustment for APOM $(\mathrm{p}=0.01)$. PCSK9 was significantly associated with per cent emphysema $(\beta=1.150,95 \%$ CI $1.0-1.32 ; \mathrm{p}=0.016)$.

\section{Association of HDL with lung function and per cent emphysema in MESA}

Among 3044 participants with spirometry, higher HDL levels were independently associated with a lower FEV1/FVC ratio $\left(-0.24 \%\right.$ per $\left.10 \mathrm{mg} \cdot \mathrm{dL}^{-1} \mathrm{HDL}, 95 \% \mathrm{CI}-0.45--0.03 ; \mathrm{p}=0.027\right)$.

Among 8367 participants with per cent emphysema data, higher HDL levels were independently associated with greater per cent emphysema $\left(0.53 \%\right.$ increase in per cent emphysema per $10 \mathrm{mg} \cdot \mathrm{dL}^{-1} \mathrm{HDL}, 95 \%$ CI $0.34-0.73 ; \mathrm{p}<0.001)$. Figure 3 shows the multivariate relationship of HDL to per cent emphysema, which was nonlinear $(\mathrm{p}<0.001)$ with a plateau at HDL levels $>60 \mathrm{mg} \cdot \mathrm{dL}^{-1}$.

To assess for potential survival bias among older participants, we repeated the HDL-emphysema analysis among 5241 participants aged 45-65 years and found consistent results. Findings were also consistent within strata of sex, race and smoking history (online supplementary material).

\section{Discussion}

This large biracial study identified two new SNPs for FEV1/FVC ratio, one in European-Americans (rs805301) and one in the combined European-American and African-American analysis (rs707974). Both SNPs were originally selected as APOM polymorphisms and are now annotated in genes flanking APOM. Both replicated in an independent sample. In addition, rs707974 was significantly associated with per cent emphysema in both European-Americans and African-Americans, APOM gene expression was associated with FEV1/FVC and per cent emphysema and HDL was associated with FEV1/FVC and per cent emphysema.

TABLE 3 Association of single nucleotide polymorphisms (SNPs) flanking APOM (apolipoprotein M) with per cent emphysema among European-American and African-American participants in the Multi-Ethnic Study of Atherosclerosis

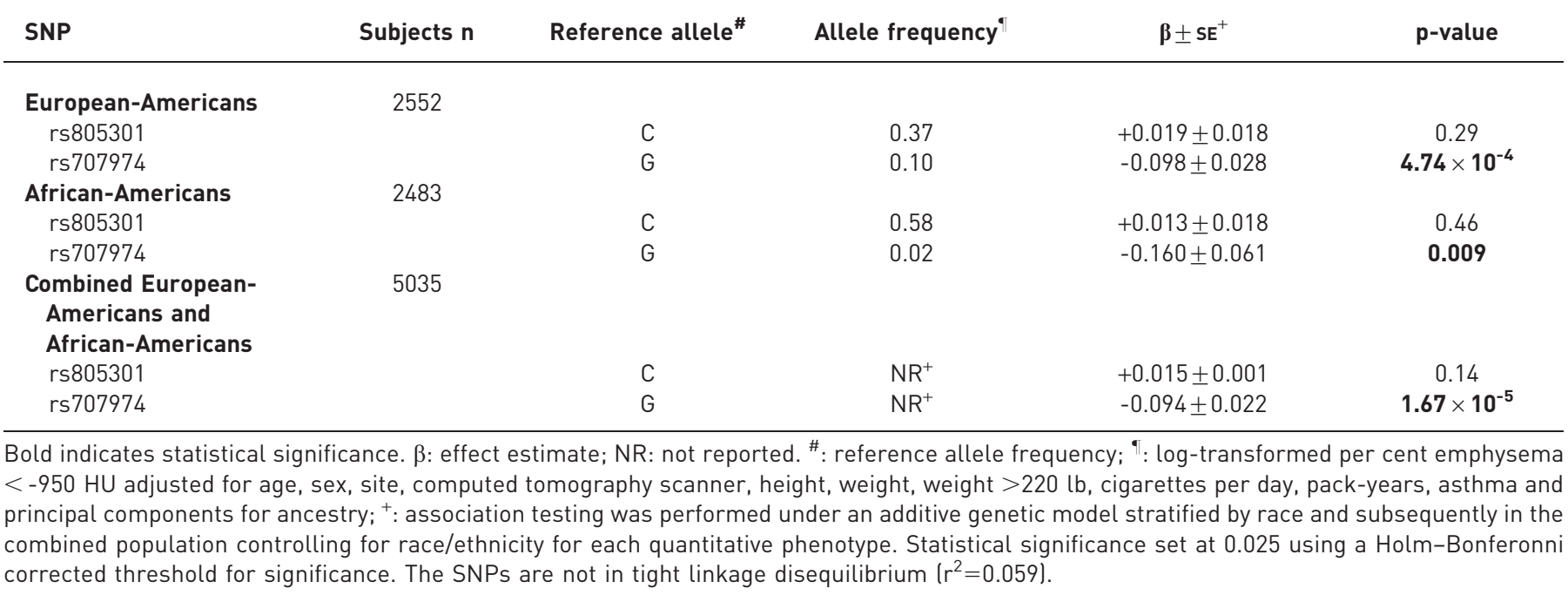


TABLE 4 Association of APOM (apolipoprotein M) gene expression with forced expiratory volume in $1 \mathrm{~s}$ (FEV1)/forced vital capacity (FVC) ratio and computed tomography (CT) per cent emphysema in 101 participants in the MESA COPD study

$\begin{array}{ccc}\begin{array}{c}\text { Difference in FEV1/FVC per increase in } \\ \text { APOM gene expression" }\end{array} & \begin{array}{c}\text { Difference in per cent emphysema per } \\ \text { increase in APOM gene expression }\end{array}\end{array}$

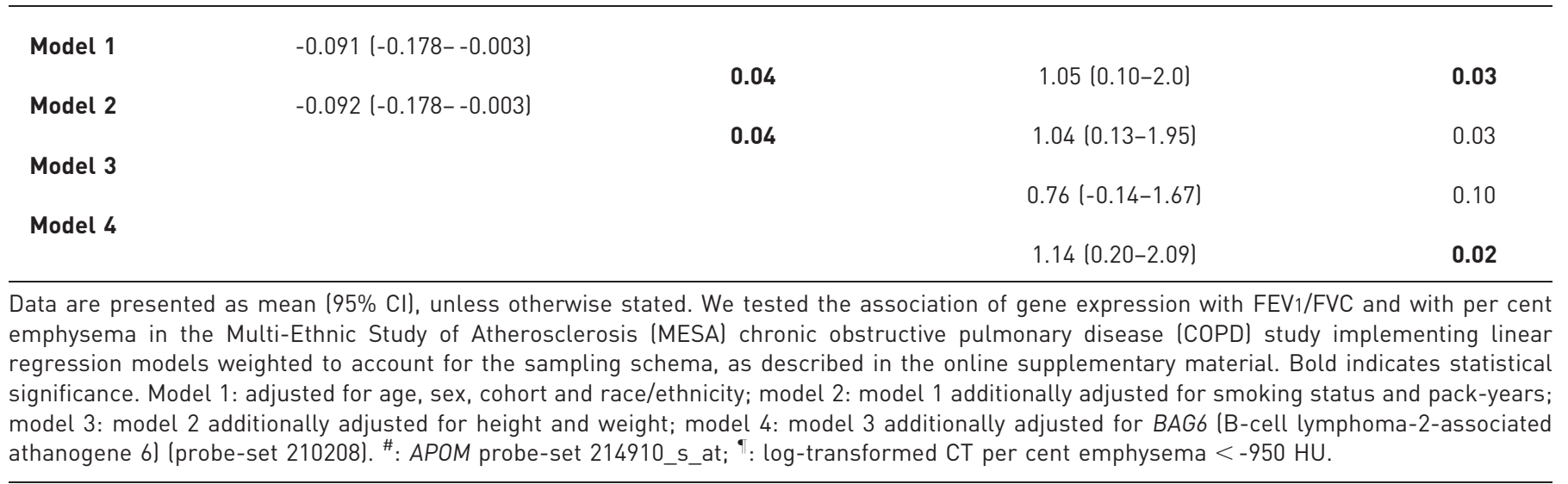

The identified SNPs flanking $A P O M$ are unlikely to be causative variants, but might be linked with a functional $A P O M$ variant. Consistent with this thinking, the APOM promoter SNP rs805297 alters APOM expression [68] and is in weak linkage disequilibrium with rs707974 among African-Americans and European-Americans $\left(\mathrm{r}^{2}=0.36\right.$ and $\mathrm{r}^{2}=0.32$, respectively) and $\mathrm{rs} 805301$ among European-Americans $\left(\mathrm{r}^{2}=0.23\right)$ (online supplementary fig S7).

APOM encodes apolipoprotein M, a lipoprotein-associated plasma protein [69]. The majority of apolipoprotein $\mathrm{M}$ is found in HDL [70]. In murine models, modifying APOM gene expression changes apolipoprotein $M$ plasma concentration, which affects HDL levels, pre- $\beta$-HDL formation, reverse cholesterol transport and remodels plasma HDL $[42,70]$. Hence, APOM gene expression alters the function and quality of HDL.

Apolipoprotein M and HDL are relevant to the pathogenesis of COPD, particularly emphysema, via three related pathways. First, HDL inhibits tumour necrosis factor-stimulated sphingosine kinase activity in human endothelial cells, thereby increasing ceramide and decreasing sphingosine-1-phosphate (S1P) cellular levels $[41,71]$. Ceramide, a second-messenger molecule, modulates endothelial cell apoptosis and is implicated in emphysema pathogenesis $[39,40]$. Secondly, HDL-associated apolipoprotein M is the plasma carrier for S1P and this HDL-apolipoprotein M subclass presents S1P to the S1P1 endothelial cell receptor, which is endothelium-protective [44,72]. S1P has an essential role in maintaining endothelial barrier integrity in the lung and is implicated in emphysema pathogenesis [44, 73]. Thirdly, HDL binds and incorporates $\alpha_{1}$-antitrypsin. HDL-bound $\alpha_{1}$-antitrypsin inhibits extracellular matrix degradation and apoptosis in vascular smooth muscle [74, 75].

The relevance of APOM and HDL to COPD pathogenesis is further reinforced by our findings that PCSK 9 polymorphisms were associated with FEV1/FVC ratio and, nominally, per cent emphysema, and that PCSK9 gene expression was associated with per cent emphysema. PCSK9 augments the degradation of low density lipoprotein receptors [76] and gain-of-function mutations in PCSK9 cause familial hypercholesterolaemia [77]. HDL levels in patients with PCSK9 mutations are generally increased [78-80] and most placebocontrolled trials of PCSK9 inhibitors have shown modest increases in HDL levels [81-85]. Furthermore, plasma levels of PCSK9 are associated with plasma apolipoprotein M levels [67].

The association of apolipoprotein M and HDL with FEV1/FVC and emphysema are probably distinct from their relationships to cardiovascular disease and we speculate that the roles of HDL and apolipoprotein M in the lungs are different from their roles in atherosclerosis. Although HDL has long been thought to be atheroprotective, the definitive epidemiological study on HDL and cardiovascular disease suggested no benefit [86] and large-scale randomised clinical trials of cholesterol ester transfer proteins, which raise HDL levels, have yet to show a benefit on clinical cardiovascular events [87, 88]. The literature on apolipoprotein $\mathrm{M}$ in cardiovascular disease is relatively small and mixed, with animal studies suggesting atheroprotective effects [42, 43]; however, in humans, plasma apolipoprotein $M$ levels were not associated with atherosclerotic disease [89]. 


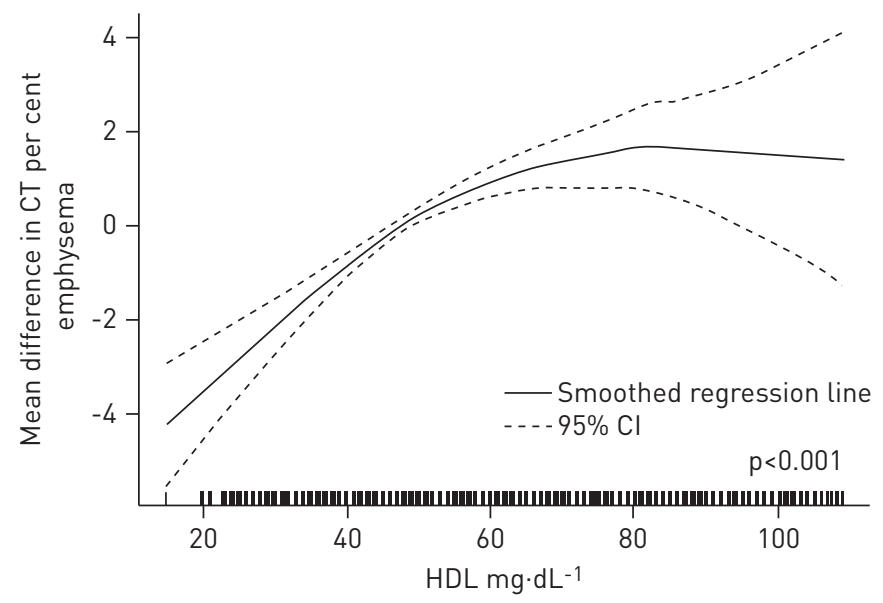

FIGURE 3 Multivariate association between high-density lipoprotein (HDL) and computed tomography (CT) per cent emphysema. Results of multivariate analyses of the relationship between per cent emphysema and plasma HDL among 8367 Multi-Ethnic Study of Atherosclerosis Single Nucleotide Polymorphism Health Association Resource participants are shown. Smoothed regression line adjusted for age, sex, race/ethnicity, height, weight, educational attainment, scanner, tube current, total cholesterol, exercise, pack-years, cigarettes per day, alcohol use, inhaled steroids and use of statins. The figure and p-value was produced using a LOESS smoothing function in a generalised additive model (GAM) in R/GAM (R version 2.13.0; The R Project for Statistical Computing www.r-project.org).

Despite the strong mechanistic support implicating APOM in COPD, the latest genome build annotates the new SNPs in genes neighbouring $A P O M$, which raises the possibility that they are unrelated to APOM. GPANK1 may be involved in immunity [90] and BAG6 is implicated in apoptosis [90]; both are associated with lung cancer $[91,92]$ and neither has been associated with cardiovascular disease. Given that genome builds change over time, the identification of the two new SNPs in the same region in separate racial groups, the gene expression findings and the HDL associations all suggest that APOM rather than the other genes are implicated in COPD pathogenesis.

Previous studies of genetic risk for emphysema include two GWAS [19,20] and candidate gene association studies [21-26]. The GWAS identified BICD1 (bicaudal D homologue 1) as associated with severe emphysema on radiologist interpretation and $\alpha$-mannosidase-related genes for per cent emphysema [19]. The candidate gene association studies did not include rs707974 or rs805301.

Two small studies found increased levels of HDL in severe COPD defined by spirometry [93, 94]. Conversely, lower HDL was associated with lower FEV1 in a population-based study (which did not report the association for FEV1/FVC ratio) [95] and with advanced COPD and emphysema patients from the Evaluation of COPD Longitudinally to Identify Predictive Surrogate End-points Study [96].

The study has several potential limitations. The genomic inflation factor for the meta-analysis in EuropeanAmericans was 1.080, suggesting possible population stratification. However, we adjusted our analysis with 10 principal components for ancestry and with cohort-specific and overall genomic control to address population stratification. Furthermore, we replicated the new APOM SNP identified in EuropeanAmericans (rs805301) in African-Americans, and both SNPs replicated in an independent cohort and with gene expression, all of which makes population stratification less of a concern.

Although we analysed two phenotypes of COPD in general population samples, these traits do not capture the entire phenotypic complexity of clinical COPD. However, results for the two phenotypes were consistent with each other, similar among patients with airflow limitation, and supported by gene expression in a study of clinical disease. Furthermore, multiple prior genes identified for lung function in population-based samples have been replicated in studies of clinical COPD [15-17]. Hence, it is likely that the current findings apply to clinical COPD.

The association between HDL and per cent emphysema may be subject to confounding and reverse causation; a small study suggested that HDL levels decrease in COPD patients undergoing lung transplantation [97]. However, we adjusted for multiple potential confounders in this well-phenotyped cohort and the genetic studies are unlikely to be subject to reverse causation.

Similar to other population-based GWAS, we used pre-bronchodilator spirometry for lung function measurement. However, per cent emphysema was measured on partial-lung CT scans; previously, we validated per cent emphysema on partial-lung scans compared to full-lung scans in this cohort and have 
confirmed multiple prior hypotheses using them [30, 59]. Per cent emphysema, like lung function, is related to sex, body size, ancestry and socioeconomic status [98], in addition to current smoking [99]. However, we adjusted for all of these variables in the analyses.

In conclusion, we identified one new SNP related to FEV1/FVC ratio among European-Americans and a second new SNP in the combined European-American and African-American analysis, which was also associated with per cent emphysema. Both new SNPs flank APOM, and APOM expression was associated with FEV1/FVC ratio and per cent emphysema. APOM encodes apolipoprotein $\mathrm{M}$, which is primarily bound to HDL, and higher levels of HDL were associated with lower FEV1/FVC ratio and greater per cent emphysema. Together, these findings suggest a novel effect of the apolipoprotein M-HDL-cholesterol pathway in the pathogenesis of COPD and emphysema. Further examination of this pathway is warranted to determine whether it could be targeted to treat or prevent COPD, and ongoing clinical trials of PCSK9inhibitors [81-85] and other medications that raise HDL levels [88] may consider monitoring for pulmonary effects.

\section{Acknowledgements}

The authors' affiliations are as follows: K.M. Burkart: Dept of Medicine, College of Physicians and Surgeons, Columbia University, New York, NY, USA; A. Manichaikul: Center for Public Health Genomics, University of Virginia School of Medicine, Charlottesville, VA, USA; J.B. Wilk: Dept of Medicine, Brigham and Women's Hospital and Harvard Medical School, Boston, MA, USA; F.S. Ahmed: Depts of Medicine and Radiology, College of Physicians and Surgeons, Columbia University, New York, NY, USA; G.L. Burke: Dept of Public Health Sciences, Wake Forest School of Medicine, WinstonSalem, NC, USA; P. Enright: Dept of Medicine, University of Arizona, Tucson, AZ, USA; N.N. Hansel: Dept of Medicine, Johns Hopkins University, Baltimore, MD, USA; D. Haynes: Dept of Medicine, University of Mississippi, Jackson, MS, USA; S.R. Heckbert: Dept of Epidemiology, School of Public Health, University of Washington, Seattle, WA, USA; E.A. Hoffman: Dept of Radiology, University of Iowa, Iowa City, IA, USA; J.D. Kaufman: Depts of Environmental and Occupational Health Sciences, Medicine, and Epidemiology, University of Washington, Seattle, WA, USA; J. Kurai: Dept of Medicine, Mount Sinai Hospital, New York, NY, USA; L. Loehr: Dept of Epidemiology, University of North Carolina, Chapel Hill, NC, USA; S.J. London: Epidemiology Branch, National Institute of Environmental Health Sciences, National Institutes of Health and Human Services, Research Triangle Park, NC, USA; Y. Meng: The Broad Institute of MIT and Harvard, Cambridge, MA, USA; G.T. O’Connor: Pulmonary Center, Dept of Medicine, Boston University School of Medicine, Boston, MA and National Heart, Lung, and Blood Institute (NHLBI) Framingham Heart Study, Framingham, MA, USA; E. Oelsner: Dept of Medicine, College of Physicians and Surgeons, Columbia University, New York, NY, USA; M. Petrini: Dept of Medicine, University of Mississippi, Jackson, MS, USA; T.D. Pottinger: Dept of Medicine, College of Physicians and Surgeons, Columbia University, New York, NY, USA; C.A. Powell: Dept of Medicine, Mount Sinai Hospital, New York, NY, USA; S. Redline: Dept of Medicine, Brigham and Women's Hospital, Beth Israel Deaconess Medical Center and Harvard Medical School, Boston, MA, USA; J.I. Rotter: Medical Genetics Institute, Cedars-Sinai Medical Center, Los Angeles, CA, USA; L.J. Smith: Dept of Medicine, Northwestern University, Chicago, IL, USA; M. Soler Artigas: Dept of Health Sciences, Genetic Epidemiology Group, University of Leicester, Leicester, UK; M.D. Tobin: Dept of Health Sciences, Genetic Epidemiology Group, University of Leicester, and National Institute for Health Research (NIHR) Leicester Respiratory Biomedical Research Unit, Glenfield Hospital, Leicester, UK; M.Y. Tsai: Dept of Laboratory Medical Pathology, University of Minnesota, Minneapolis, MN, USA; K. Watson: Dept of Medicine, University of California Los Angeles, Los Angeles, CA, USA; W. White: Jackson Heart Study, Tougaloo College, Tougaloo, MS, USA; T.R. Young: Broad Institute of Massachusetts Institute of Technology and Harvard University, Cambridge, MA, USA; S.S. Rich: Center for Public Health Genomics, University of Virginia School of Medicine, Charlottesville, VA, USA; R.G. Barr: Dept of Medicine, College of Physicians and Surgeons and Dept of Epidemiology, Mailman School of Public Health, Columbia University, New York, NY, USA.

We wish to acknowledge the support of the NHLBI and the contributions of the research institutions, study investigators, field staff and study participants in creating this resource for biomedical research.

The following parent studies have contributed parent study data, ancillary study data and DNA samples through the Broad Institute (N01-HC-65226) to create this genotype/phenotype database for wide dissemination to the biomedical research community: Atherosclerosis Risk in Communities (ARIC), Coronary Artery Risk Development in Young Adults (CARDIA), Cleveland Family Study (CFS), Cardiovascular Health Study (CHS), Framingham Heart Study (FHS), Jackson Heart Study (JHS), Multi-Ethnic Study of Atherosclerosis (MESA), MESA Family, MESA Air Pollution and MESA Lung studies. A full list of participating MESA investigators and institutions can be found at www.mesa-nhlbi.org. A full list of principal CHS investigators and institutions can be found at www.chs-nhlbi.org. This manuscript has been reviewed by the MESA investigators for scientific content and consistency of data interpretation with previous MESA publications and significant comments have been incorporated prior to submission for publication.

The ARIC study is performed as a collaborative study supported by NHLBI. We thank the staff and participants of the ARIC study for their important contributions.

We thank the research institutions, study investigators, field staff and study participants for the following studies which contributed to SpiroMeta consortium Avon Longitudinal Study of Parents and Children, B58C-T1DGC, B58C-WTCCC, European Prospective Investigation of Cancer (EPIC) obese cases, EPIC population based, Academy of Finland Centre of Excellence in Complex Disease Genetics (FTC), Cooperative health research in the Region of Augsburg (KORA) S3, the Korcula study, Northern Finland Birth Cohort (NFBC) 1966, Northern Swedish Population Health Study (NSPHS), Orkney Complex Disease Study (ORCADES), Study of Health in Pomerania, the Twins UK study and the Vis study. 


\section{References}

1 Lozano R, Naghavi M, Foreman K, et al. Global and regional mortality from 235 causes of death for 20 age groups in 1990 and 2010: a systematic analysis for the Global Burden of Disease Study 2010. Lancet 2012; 380: 2095-2128.

2 Petty TL, Weinmann GG. Building a national strategy for the prevention and management of and research in chronic obstructive pulmonary disease. National Heart, Lung, and Blood Institute Workshop Summary. Bethesda, Maryland, August 29-31, 1995. JAMA 1997; 277: 246-253.

3 Pauwels RA, Buist AS, Calverley PM, et al. Global strategy for the diagnosis, management, and prevention of chronic obstructive pulmonary disease. NHLBI/WHO Global Initiative for Chronic Obstructive Lung Disease (GOLD) Workshop summary. Am J Respir Crit Care Med 2001; 163: 1256-1276.

4 McLean KH. The pathogenesis of pulmonary emphysema. Am J Med 1958; 25: 62-74.

5 Silverman EK, Chapman HA, Drazen JM, et al. Genetic epidemiology of severe, early-onset chronic obstructive pulmonary disease. Risk to relatives for airflow obstruction and chronic bronchitis. Am J Respir Crit Care Med 1998; 157: 1770-1778.

6 Silverman EK, Palmer LJ, Mosley JD, et al. Genomewide linkage analysis of quantitative spirometric phenotypes in severe early-onset chronic obstructive pulmonary disease. Am J Hum Genet 2002; 70: 1229-1239.

7 McCloskey SC, Patel BD, Hinchliffe SJ, et al. Siblings of patients with severe chronic obstructive pulmonary disease have a significant risk of airflow obstruction. Am J Respir Crit Care Med 2001; 164: 1419-1424.

8 Cho MH, McDonald M-LN, Zhou X, et al. Risk loci for chronic obstructive pulmonary disease: a genome-wide association study and meta-analysis. Lancet Respir Med 2014 [In press DOI: 10.1016/S2213-2600(14)70002-5].

9 Soler Artigas M, Loth DW, Wain LV, et al. Genome-wide association and large-scale follow up identifies 16 new loci influencing lung function. Nat Genet 2011; 43: 1082-1090.

10 Wilk JB, Shrine NR, Loehr LR, et al. Genome-wide association studies identify CHRNA5/3 and HTR4 in the development of airflow obstruction. Am J Respir Crit Care Med 2012; 186: 622-632.

11 Hancock DB, Soler Artigas M, Gharib SA, et al. Genome-wide joint meta-analysis of SNP and SNP-by-smoking interaction identifies novel loci for pulmonary function. PLoS Genet 2012; 8: e1003098.

12 Repapi E, Sayers I, Wain LV, et al. Genome-wide association study identifies five loci associated with lung function. Nat Genet 2010; 42: 36-44.

13 Hancock DB, Eijgelsheim M, Wilk JB, et al. Meta-analyses of genome-wide association studies identify multiple loci associated with pulmonary function. Nat Genet 2010; 42: 45-52.

14 Wilk JB, Chen TH, Gottlieb DJ, et al. A genome-wide association study of pulmonary function measures in the Framingham Heart Study. PLoS Genet 2009; 5: e1000429.

15 Cho MH, Boutaoui N, Klanderman BJ, et al. Variants in FAM13A are associated with chronic obstructive pulmonary disease. Nat Genet 2010; 42: 200-202.

16 Soler Artigas M, Wain LV, Repapi E, et al. Effect of five genetic variants associated with lung function on the risk of chronic obstructive lung disease, and their joint effects on lung function. Am J Respir Crit Care Med 2011; 184: 786-795.

17 Castaldi PJ, Cho MH, Litonjua AA, et al. The association of genome-wide significant spirometric loci with chronic obstructive pulmonary disease susceptibility. Am J Respir Cell Mol Biol 2011; 45: 1147-1153.

18 Patel BD, Coxson HO, Pillai SG, et al. Airway wall thickening and emphysema show independent familial aggregation in chronic obstructive pulmonary disease. Am J Respir Crit Care Med 2008; 178: 500-505.

19 Kong X, Cho MH, Anderson W, et al. Genome-wide association study identifies BICD1 as a susceptibility gene for emphysema. Am J Respir Crit Care Med 2011; 183: 43-49.

20 Manichaikul A, Hoffman EA, Smolonska J, et al. Genome-wide Study of Percent Emphysema on CT in the General Population: The MESA Lung/SHARe Study. Am J Respir Crit Care Med 2014 [In press DOI: 10.1164/rccm.201306$10610 \mathrm{C}]$.

21 DeMeo DL, Hersh CP, Hoffman EA, et al. Genetic determinants of emphysema distribution in the National Emphysema Treatment Trial. Am J Respir Crit Care Med 2007; 176: 42-48.

22 Hersh CP, Hansel NN, Barnes KC, et al. Transforming growth factor- $\beta$ receptor-3 is associated with pulmonary emphysema. Am J Respir Cell Mol Biol 2009; 41: 324-331.

23 Lambrechts D, Buysschaert I, Zanen P, et al. The 15q24/25 susceptibility variant for lung cancer and chronic obstructive pulmonary disease is associated with emphysema. Am J Respir Crit Care Med 2010; 181: 486-493.

24 Kim DK, Hersh C, Washko GR, et al. Epidemiology, radiology, and genetics of nicotine dependence in COPD. Respir Res 2011; 12: 9.

25 Yamada M, Ishii T, Ikeda S, et al. Association of fucosyltransferase 8 (FUT8) polymorphism Thr267Lys with pulmonary emphysema. J Hum Genet 2011; 56: 857-860.

26 Ishii T, Hagiwara K, Ikeda S, et al. Association between genetic variations in surfactant protein D and emphysema, interstitial pneumonia, and lung cancer in a Japanese population. COPD 2012; 9: 409-416.

27 Keating BJ, Tischfield S, Murray SS, et al. Concept, design and implementation of a cardiovascular gene-centric $50 \mathrm{k}$ SNP array for large-scale genomic association studies. PLoS One 2008; 3: e3583.

28 Giordano RJ, Lahdenranta J, Zhen L, et al. Targeted induction of lung endothelial cell apoptosis causes emphysema-like changes in the mouse. J Biol Chem 2008; 283: 29447-29460.

29 Clauss M, Voswinckel R, Rajashekhar G, et al. Lung endothelial monocyte-activating protein 2 is a mediator of cigarette smoke-induced emphysema in mice. J Clin Invest 2011; 121: 2470-2479.

30 Barr RG, Bluemke DA, Ahmed FS, et al. Percent emphysema, airflow obstruction, and impaired left ventricular filling. N Engl J Med 2010; 362: 217-227.

31 Barr RG, Ahmed FS, Carr JJ, et al. Subclinical atherosclerosis, airflow obstruction and emphysema: the MESA Lung Study. Eur Respir J 2012; 39: 846-854.

32 Kim JA, Montagnani M, Koh KK, et al. Reciprocal relationships between insulin resistance and endothelial dysfunction: molecular and pathophysiological mechanisms. Circulation 2006; 113: 1888-1904.

33 Kasahara Y, Tuder RM, Cool CD, et al. Endothelial cell death and decreased expression of vascular endothelial growth factor and vascular endothelial growth factor receptor 2 in emphysema. Am J Respir Crit Care Med 2001; 163: 737-744. 

arteries of smokers and patients with moderate chronic obstructive pulmonary disease. Am J Respir Crit Care Med 2003; 167: 1250-1256.

35 Kanazawa H, Asai K, Hirata K, et al. Possible effects of vascular endothelial growth factor in the pathogenesis of chronic obstructive pulmonary disease. Am J Med 2003; 114: 354-358.

36 Leibow AA. Pulmonary emphysema with special reference to vascular changes. Am Rev Respir Dis 1959; 80: 67-93.

37 Barr RG, Mesia-Vela S, Austin $\mathrm{JH}$, et al. Impaired flow-mediated dilation is associated with low pulmonary function and emphysema in ex-smokers: the Emphysema and Cancer Action Project (EMCAP) Study. Am J Respir Crit Care Med 2007; 176: 1200-1207.

38 Tuder RM, Petrache I, Elias JA, et al. Apoptosis and emphysema: the missing link. Am J Respir Cell Mol Biol 2003; 28: 551-554.

39 Tuder RM, Zhen L, Cho CY, et al. Oxidative stress and apoptosis interact and cause emphysema due to vascular endothelial growth factor receptor blockade. Am J Respir Cell Mol Biol 2003; 29: 88-97.

40 Petrache I, Natarajan V, Zhen L, et al. Ceramide upregulation causes pulmonary cell apoptosis and emphysema-like disease in mice. Nat Med 2005; 11: 491-498.

41 Xia P, Vadas MA, Rye KA, et al. High density lipoproteins (HDL) interrupt the sphingosine kinase signaling pathway. A possible mechanism for protection against atherosclerosis by HDL. J Biol Chem 1999; 274: 33143-33147.

42 Wolfrum C, Poy MN, Stoffel M. Apolipoprotein M is required for pre- $\beta$-HDL formation and cholesterol efflux to HDL and protects against atherosclerosis. Nat Med 2005; 11: 418-422.

43 Christoffersen C, Jauhiainen M, Moser M, et al. Effect of apolipoprotein M on high density lipoprotein metabolism and atherosclerosis in low density lipoprotein receptor knock-out mice. J Biol Chem 2008; 283: $1839-1847$.

44 Christoffersen C, Obinata H, Kumaraswamy SB, et al. Endothelium-protective sphingosine-1-phosphate provided by HDL-associated apolipoprotein M. Proc Natl Acad Sci USA 2011; 108: 9613-9618.

45 Musunuru K, Lettre G, Young T, et al. Candidate gene association resource (CARe): design, methods, and proof of concept. Circ Cardiovasc Genet 2010; 3: 267-275.

46 Bild DE, Bluemke DA, Burke GL, et al. Multi-ethnic study of atherosclerosis: objectives and design. Am J Epidemiol 2002; 156: 871-881.

47 The ARIC investigators. The Atherosclerosis Risk in Communities (ARIC) Study: design and objectives. Am $J$ Epidemiol 1989; 129: 687-702.

48 Fried LP, Borhani NO, Enright P, et al. The Cardiovascular Health Study: design and rationale. Ann Epidemiol 1991; 1: 263-276.

49 Dawber TR, Kannel WB. The Framingham study. An epidemiological approach to coronary heart disease. Circulation 1966; 34: 553-555.

50 Feinleib M, Kannel WB, Garrison RJ, et al. The Framingham Offspring Study. Design and preliminary data. Prev Med 1975; 4: 518-525.

51 Friedman GD, Cutter GR, Donahue RP, et al. CARDIA: study design, recruitment, and some characteristics of the examined subjects. J Clin Epidemiol 1988; 41: 1105-1116.

52 Buxbaum SG, Elston RC, Tishler PV, et al. Genetics of the apnea hypopnea index in Caucasians and African Americans: I. Segregation analysis. Genet Epidemiol 2002; 22: 243-253.

53 Taylor HA Jr. The Jackson Heart Study: an overview. Ethn Dis 2005; 15: Suppl. 6, 1-3.

54 Carpenter MA, Crow R, Steffes M, et al. Laboratory, reading center, and coordinating center data management methods in the Jackson Heart Study. Am J Med Sci 2004; 328: 131-144.

55 Rodriguez J, Jiang R, Johnson WC, et al. The association of pipe and cigar use with cotinine levels, lung function, and airflow obstruction: a cross-sectional study. Ann Intern Med 2010; 152: 201-210.

56 Hankinson JL, Odencrantz JR, Fedan KB. Spirometric reference values from a sample of the general U.S. population. Am J Respir Crit Care Med 1999; 159: 179-187.

57 Manichaikul A, Chen WM, Williams K, et al. Analysis of family- and population-based samples in cohort genomewide association studies. Hum Genet 2012; 131: 275-287.

58 Kaufman JD, Adar SD, Allen RW, et al. Prospective study of particulate air pollution exposures, subclinical atherosclerosis, and clinical cardiovascular disease: The Multi-Ethnic Study of Atherosclerosis and Air Pollution (MESA Air). Am J Epidemiol 2012; 176: 825-837.

59 Hoffman EA, Jiang R, Baumhauer H, et al. Reproducibility and validity of lung density measures from cardiac CT scans - the Multi-Ethnic Study of Atherosclerosis (MESA) Lung Study. Acad Radiol 2009; 16: 689-699.

60 Tsai MY, Johnson C, Kao WHL, et al. Cholesteryl ester transfer protein genetic polymorphisms, HDL cholesterol, and subclinical cardiovascular disease in the Multi-Ethnic Study of Atherosclerosis. Atherosclerosis 2008; 200: 359-367.

61 Purcell S, Neale B, Todd-Brown K, et al. PLINK: a tool set for whole-genome association and population-based linkage analyses. Am J Hum Genet 2007; 81: 559-575.

62 Chen MH, Yang Q. GWAF: an R package for genome-wide association analyses with family data. Bioinformatics 2010; 26: 580-581.

63 Price AL, Patterson NJ, Plenge RM, et al. Principal components analysis corrects for stratification in genome-wide association studies. Nat Genet 2006; 38: 904-909.

64 Willer CJ, Li Y, Abecasis GR. METAL: fast and efficient meta-analysis of genomewide association scans. Bioinformatics 2010; 26: 2190-2191.

65 Holm S. A simple sequentially rejective multiple test procedure. Scand J Stat 1979; 6: 65-70.

66 Pruim RJ, Welch RP, Sanna S, et al. LocusZoom: regional visualization of genome-wide association scan results. Bioinformatics 2010; 26: 2336-2337.

67 Kappelle PJ, Lambert G, Dahlbäck B, et al. Relationship of plasma apolipoprotein M with proprotein convertase subtilisin-kexin type 9 levels in non-diabetic subjects. Atherosclerosis 2011; 214: 492-494.

$68 \mathrm{Hu}$ HJ, Jin EH, Yim SH, et al. Common variants at the promoter region of the APOM confer a risk of rheumatoid arthritis. Exp Mol Med 2011; 43: 613-621.

69 Xu N, Dahlbäck B. A novel human apolipoprotein (apoM). J Biol Chem 1999; 274: 31286-31290. 
70 Nielsen LB, Christoffersen C, Ahnström J, et al. ApoM: gene regulation and effects on HDL metabolism. Trends Endocrinol Metab 2009; 20: 66-71.

71 Xia P, Gamble JR, Rye KA, et al. Tumor necrosis factor- $\alpha$ induces adhesion molecule expression through the sphingosine kinase pathway. Proc Natl Acad Sci USA 1998; 95: 14196-14201.

72 Karuna R, Park R, Othman A, et al. Plasma levels of sphingosine-1-phosphate and apolipoprotein M in patients with monogenic disorders of HDL metabolism. Atherosclerosis 2011; 219: 855-863.

73 Diab KJ, Adamowicz JJ, Kamocki K, et al. Stimulation of sphingosine 1-phosphate signaling as an alveolar cell survival strategy in emphysema. Am J Respir Crit Care Med 2010; 181: 344-352.

74 Karlsson H, Leanderson P, Tagesson C, et al. Lipoproteomics II: mapping of proteins in high-density lipoprotein using two-dimensional gel electrophoresis and mass spectrometry. Proteomics 2005; 5: 1431-1445.

75 Ortiz-Muñoz G, Houard X, Martín-Ventura JL, et al. HDL antielastase activity prevents smooth muscle cell anoikis, a potential new antiatherogenic property. FASEB J 2009; 23: 3129-3139.

76 Holla ØL, Laerdahl JK, Strøm TB, et al. Removal of acidic residues of the prodomain of PCSK9 increases its activity towards the LDL receptor. Biochem Biophys Res Commun 2011; 406: 234-238.

77 Abifadel M, Varret M, Rabès JP, et al. Mutations in PCSK9 cause autosomal dominant hypercholesterolemia. Nat Genet 2003; 34: 154-156.

78 Abifadel M, Rabès JP, Devillers M, et al. Mutations and polymorphisms in the proprotein convertase subtilisin kexin 9 (PCSK9) gene in cholesterol metabolism and disease. Hum Mutat 2009; 30: 520-529.

79 Aung LH, Yin RX, Miao L, et al. The proprotein convertase subtilisin/kexin type 9 gene E670G polymorphism and serum lipid levels in the Guangxi Bai Ku Yao and Han populations. Lipids Health Dis 2011; 10: 5.

80 Cohen JC, Boerwinkle E, Mosley TH Jr, et al. Sequence variations in PCSK9, low LDL, and protection against coronary heart disease. N Engl J Med 2006; 354: 1264-1272.

81 Sullivan D, Olsson AG, Scott R, et al. Effect of a monoclonal antibody to PCSK9 on low-density lipoprotein cholesterol levels in statin-intolerant patients: the GAUSS randomized trial. JAMA 2012; 308: 2497-2506.

82 Raal F, Scott R, Somaratne R, et al. Low-density lipoprotein cholesterol-lowering effects of AMG 145, a monoclonal antibody to proprotein convertase subtilisin/kexin type 9 serine protease in patients with heterozygous familial hypercholesterolemia: the Reduction of LDL-C with PCSK9 Inhibition in Heterozygous Familial Hypercholesterolemia Disorder (RUTHERFORD) randomized trial. Circulation 2012; 126: 2408-2417.

83 Giugliano RP, Desai NR, Kohli P, et al. Efficacy, safety, and tolerability of a monoclonal antibody to proprotein convertase subtilisin/kexin type 9 in combination with a statin in patients with hypercholesterolaemia (LAPLACETIMI 57): a randomised, placebo-controlled, dose-ranging, phase 2 study. Lancet 2012; 380: 2007-2017.

84 Roth EM, McKenney JM, Hanotin C, et al. Atorvastatin with or without an antibody to PCSK9 in primary hypercholesterolemia. N Engl J Med 2012; 367: 1891-1900.

85 Dias CS, Shaywitz AJ, Wasserman SM, et al. Effects of AMG 145 on low-density lipoprotein cholesterol levels: results from 2 randomized, double-blind, placebo-controlled, ascending-dose phase 1 studies in healthy volunteers and hypercholesterolemic subjects on statins. J Am Coll Cardiol 2012; 60: 1888-1898.

86 Voight BF, Peloso GM, Orho-Melander M, et al. Plasma HDL cholesterol and risk of myocardial infarction: a mendelian randomisation study. Lancet 2012; 380: 572-580.

87 Barter PJ, Caulfield M, Eriksson M, et al. Effects of torcetrapib in patients at high risk for coronary events. $N$ Engl $J$ Med 2007; 357: 2109-2122.

88 Schwartz GG, Olsson AG, Abt M, et al. Effects of dalcetrapib in patients with a recent acute coronary syndrome. N Engl J Med 2012; 367: 2089-2099.

89 Ahnström J, Axler O, Jauhiainen M, et al. Levels of apolipoprotein M are not associated with the risk of coronary heart disease in two independent case-control studies. J Lipid Res 2008; 49: 1912-1917.

90 Pruitt KD, Tatusova T, Klimke W, et al. NCBI Reference Sequences: current status, policy and new initiatives. Nucleic Acids Res 2009; 37: D32-D36.

91 Rudd MF, Webb EL, Matakidou A, et al. Variants in the GH-IGF axis confer susceptibility to lung cancer. Genome Res 2006; 16: 693-701.

92 Wang Y, Broderick P, Webb E, et al. Common 5p15.33 and 6p21.33 variants influence lung cancer risk. Nat Genet 2008; 40: 1407-1409.

93 Tisi GM, Conrique A, Barrett-Connor E, et al. Increased high density lipoprotein cholesterol in obstructive pulmonary disease (predominant emphysematous type). Metabolism 1981; 30: 340-346.

94 Reed RM, Iacono A, DeFilippis A, et al. Advanced chronic obstructive pulmonary disease is associated with high levels of high-density lipoprotein cholesterol. J Heart Lung Transplant 2011; 30: 674-678.

95 Cirillo DJ, Agrawal Y, Cassano PA. Lipids and pulmonary function in the Third National Health and Nutrition Examination Survey. Am J Epidemiol 2002; 155: 842-848.

96 Ubhi BK, Riley JH, Shaw PA, et al. Metabolic profiling detects biomarkers of protein degradation in COPD patients. Eur Respir J 2012; 40: 345-355.

97 Reed RM, Hashmi S, Eberlein M, et al. Impact of lung transplantation on serum lipids in COPD. Respir Med 2011; 105: 1961-1968.

98 Lovasi GS, Diez Roux AV, Hoffman EA, et al. Socioeconomic status is positively associated with percent emphysema on CT scan: the MESA lung study. Acad Radiol 2011; 18: 199-204.

99 Ashraf H, Lo P, Shaker SB, et al. Short-term effect of changes in smoking behaviour on emphysema quantification by CT. Thorax 2011; 66: 55-60. 